\title{
NECESIDADES FORMATIVAS DE LOS CUIDADORES INMIGRANTES DE PERSONAS MAYORES EN EL HOGAR EN EUROPA: PROYECTO IENE7
}

\author{
Patricia Rocamora Pérez \\ rocamora@ual.es \\ Remedios López Liria \\ rll040@ual.es \\ David Padilla Góngora \\ dpadilla@ual.es \\ Ana Morales Montoya \\ moralesmontoyaana@gmail.com \\ Pilar Díaz López \\ pilardiaz@crecimientohumano.com \\ José Manuel Aguilar Parra \\ jmaguilar@ual.es \\ Universidad de Almería
}

Fecha de Recepción: 28 Marzo 2018

Fecha de Admisión: 10 Abril 2018

\section{RESUMEN}

Introducción: El proyecto europeo IENE7 aglutina cinco socios (dos en Italia, Reino Unido, Rumanía y el grupo de investigación HUM-498 Intervención Psicológica en Desarrollo, Educación y Orientación de la Universidad de Almería) con el objetivo común de desarrollar un modelo adecuado de educación dirigido a cuidadores informales de personas mayores que reciben atención en el hogar. Este colectivo de cuidadores procede en un alto porcentaje de grupos desfavorecidos (inmigrantes, personas sin formación 0 en riesgo de exclusión social, etc.). El objetivo principal de esta fase del proyecto es conocer las necesidades de cuidado de las personas mayores en el hogar, así como las necesidades formativas de sus cuidadores, en este contexto europeo en el que se desarrolla el proyecto. Metodología: Se realizó una revisión de la literatura científica tanto a nivel nacional (España, Italia, Reino Unido y Rumanía) como internacional. Resultados: El análisis de necesidades, desde nuestra doble perspectiva de partida (necesidades de cuidado para las personas mayores y necesidades formativas para sus cuidadores informales) puede sintetizarse en seis aspectos clave: 1) Soledad, 2) Problemas de comunicación, 3) Necesidad de un cuidado centrado en la persona y culturalmente apropiado, 4) Limitación de la Movilidad, 5) Seguridad y Protección (aspectos 


\section{NECESIDADES FORMATIVAS DE LOS CUIDADORES INMIGRANTES DE PERSONAS MAYORES EN EL HOGAR EN EUROPA: PROYECTO IENE7}

éticos y legales del cuidado), 6) Recursos sociosanitarios. Conclusiones: El conocimiento de estas necesidades de cuidado para las personas mayores y formativas de sus cuidadores informales constituye un punto de partida imprescindible para la propuesta y desarrollo de un modelo adecuado de educación dirigido a este colectivo de cuidadores, capaz de dar respuesta a las mismas, objetivo general de nuestro proyecto europeo IENE7, para atender esta importante y urgente demanda social.

Palabras clave: cuidadores informales; inmigrantes; personas mayores; formación; atención en el hogar

\section{ABSTRACT} ject.

Training needs for immigrant caregivers of older people in the home in europe: iene7 pro-

Introduction: The European project IENE7 brings together five partners (two in Italy, the United Kingdom, Romania and the research group HUM-498 Psychological Intervention in Development, Education and Guidance of the University of Almeria) with the common objective of developing an appropriate model of education aimed at informal caregivers of the older people who receive care at home. This collective of caregivers comes in a high percentage from disadvantaged groups (immigrants, people without education or at risk of social exclusion, etc.). The main objective in this phase of the project is to get to know the care needs of the older people in the home, as well as the training needs of their caregivers, in this European context in which the project is developed. Methodology: A review of the scientific literature was carried out both nationally (Spain, Italy, United Kingdom and Romania) and internationally. Results: The analysis of needs, from our double perspective (care needs for the older people and training needs for their informal caregivers) can be summarized in six key aspects: 1) Loneliness, 2) Communication problems, 3) Person-centred and culturally appropriate care, 4) Restricted mobility, 5) Safeguarding issues (ethical and legal aspects of care), 6) Social and health resources. Conclusions: The knowledge of these needs of care of the older people and the needs of training of their informal caregivers is an essential starting point for the proposal and development of an appropriate model of education aimed at this group of caregivers, capable of responding to them, which constitutes the general objective of our European project IENE7, to address this important and urgent social demand.

Keywords: informal caregivers; immigrants; older people; training; home care

\section{INTRODUCCIÓN}

Los cambios en las condiciones de vida de las sociedades industrializadas está aumentando nuestra esperanza de vida (JIFE, 2015). El envejecimiento de la población es una tendencia a largo plazo, que tuvo su comienzo hace varias décadas en Europa (IENE Project, 2017).

Este incremento de la población mayor presenta implicaciones que trascienden el ámbito estrictamente demográfico, tocando el sociosanitario y el económico, como es el caso del aumento de las personas mayores dependientes y los cuidados que precisan (JIFE, 2015; Moreno-Cámara, Palomino-Moral, Moral-Fernández, Frías-Osuna \& Del-Pino-Casado, 2016; Rodríguez Rodríguez et al., 2010).

Se entiende por persona dependiente "aquella que por motivos de edad, enfermedad o discapacidad, y ligadas a la pérdida de autonomía física, sensorial, mental o intelectual, precisa con carácter permanente la atención de otra persona 0 ayuda para realizar las actividades básicas de la vida diaria" (Ley 39/2006, de 14 de diciembre). Se prevé que la tasa de dependencia de la UE-28 aumentará del 28,8\% en 2015 al 51,0\% en 2080 (Eurostat, junio de 2016).

Se entiende por atención domiciliaria "los cuidados integrales e integrados sociales y de salud, que se prestan en el domicilio a aquellas personas que por su situación de enfermedad o discapa- 
cidad, no pueden desplazarse al Centro de Salud" (Navalpotro et al., 2006). Se ha reiterado en la literatura que las necesidades asistenciales de las personas mayores dependientes no están actualmente cubiertas plenamente por el sistema sanitario $\mathrm{y} / 0$ social, de manera que esta ayuda proviene principalmente de los miembros de la misma familia o cuidadores informales que son contratados por ésta (JIFE, 2015), recurriendo principalmente a la contratación de mujeres inmigrantes para que asuman los cuidados familiares (Galiana-Gómez, de la Cuesta-Benjumea \& Donet-Montagut, 2008; JIFE, 2015).

Proporcionar atención a largo plazo en el hogar, está demostrando ser una alternativa viable y rentable al cuidado institucional. Sin embargo, debido al creciente número de adultos mayores y a la demanda de cuidadores en toda Europa, existe una escasez actual y posible de futuros cuidadores capacitados para el adecuado desempeño de su labor, lo que puede poner en riesgo esta atención en el hogar (IENE Project, 2017).

En muchos Estados miembros de la UE, son personas inmigrantes las que están cubriendo este "vacío", proporcionando servicios de atención esenciales. En 2005, a nivel europeo, el 86\% de los trabajadores que actuaban como cuidadores informales en el hogar eran extranjeros, en su mayoría empleados para el cuidado de personas mayores en el hogar (European Commission, 2009).

Estos cuidadores informales no sólo proporcionan "cuidados básicos como la higiene, alimentación y vestido, sino que son los responsables de mantener la autonomía del anciano en el hogar y de su permanencia en el entorno familiar", convirtiéndose además a menudo en el soporte afectivo más importante para el anciano (Zabalegui Yárnoz et al., 2008).

El cuidado profesional exige de un aprendizaje formal que se adquiere a través de un proceso de formación reglada (Notivol Tejero, Pollan Rufo \& Gabari Gambarte, 2004). Se debe tener en cuenta, además, que cada cultura tiene creencias, valores y pautas de cuidar y de curar propias y específicas. En este sentido, Casado-Mejia et al. (2009) encontraron un problema de definición del tipo de cuidado que se quiere obtener al contratar a una cuidadora inmigrante.

Este colectivo de cuidadores inmigrantes se enfrenta a retos importantes de educación y formación inadecuadas, salarios bajos, falta de reconocimiento de derechos laborales, falta de oportunidades de ascenso profesional y empleo irregular, además a menudo a tiempo parcial.

La falta de formación, o la inadecuación de la misma, constituye una limitación mayor. En la actualidad, no existe una regulación consistente entre todos los países, ni estándares nacionales para la formación de cuidadores de personas mayores en el hogar.

La mayoría de los currículos existentes enfocados a la formación de estos cuidadores suelen limitarse al ámbito institucional (no del hogar), y enfocarse a las habilidades para las actividades de la vida diaria, obviando otras necesidades, como las comunicativas y de relación social.

No obstante lo anterior, a menudo, la falta de éxito laboral de estos cuidadores inmigrantes se debe más específicamente a la falta de comprensión de la psicología de las personas mayores, y a la dificultad para establecer una comunicación positiva, en un ambiente amistoso, con una interacción y relación positiva con la persona mayor.

Finalmente, el cuidador inmigrante precisa igualmente conocer el sistema sociosanitario, la normativa legal y la cultura del cuidado de su nuevo lugar de trabajo, para una adaptación efectiva al nuevo ámbito sociocultural (IENE Project, 2017).

Intentando aportar soluciones a esta importante y urgente necesidad sociosanitaria, el proyecto europeo IENE7 - "Improving communication, intercultural and social skills for foreigners and migrants who work as caregivers of elderly people in Europe (ID: 2017-1-R001-KA202-037272)", aglutina cinco socios: dos en Italia, Reino Unido, Rumanía y el grupo de investigación HUM-498 Intervención Psicológica en Desarrollo, Educación y Orientación de la Universidad de Almería, con 


\section{NECESIDADES FORMATIVAS DE LOS CUIDADORES INMIGRANTES DE PERSONAS MAYORES EN EL HOGAR EN EUROPA: PROYECTO IENE7}

el objetivo común de desarrollar un modelo adecuado de educación dirigido a cuidadores informales de personas mayores que reciben atención en el hogar. Este colectivo de cuidadores procede en un alto porcentaje de grupos desfavorecidos: inmigrantes, personas sin formación 0 en riesgo de exclusión social, etc.

\section{OBJETIVOS}

El objetivo principal de esta fase del proyecto es conocer las necesidades de cuidado de las personas mayores en el hogar, así como las necesidades formativas de sus cuidadores, en este contexto europeo en el que se desarrolla el proyecto.

\section{METODOLOGÍA}

Se realizó una revisión de la literatura científica tanto a nivel nacional (España, Italia, Reino Unido y Rumanía) como internacional, dentro de las principales bases de datos sociosanitarias.

Se emplearon los siguientes descriptores (y operadores booleanos) relacionados con el objeto de esta investigación: "(Elderly[TIAB] OR Dependency[TIAB]) AND (Caregivers[TIAB] OR "Emigrants and Immigrants"[TIAB] OR "Home Care Services"[TIAB])", y por otro lado, "(Elderly OR Dependency) AND (Caregivers OR Emigrants and Immigrants OR Home Care Services)".

Para la selección de los trabajos se revisaron en primer lugar el título y resumen, realizando el primer filtro en base al objetivo del estudio. A continuación, tras obtener el texto completo, se seleccionaron sólo aquellos que cumplieron los siguientes criterios de inclusión y exclusión descritos para esta revisión:

- Criterios de inclusión: aquellas publicaciones científicas halladas a través de los descriptores, donde los pacientes fueran dependientes y/o de edad mayor 0 igual a 65 años, que permaneciesen en el ámbito domiciliario recibiendo cuidados.

- Criterios de exclusión: cuidados domiciliarios dirigidos a jóvenes o niños; atención dispensada en instituciones sanitarias o sociales; no abordaje del tema de la inmigración.

Finalmente se practicó una búsqueda en abanico, a partir de la bibliografía más relevante hallada.

\section{RESULTADOS}

En este apartado se expone de manera sintetizada el análisis de resultados integrados a partir de las búsquedas desarrolladas en paralelo, a nivel internacional y nacionales: España (Álvarez, Elordui, Errami, Rodríguez, Riguera, de la Hoz \& Márquez, 2009; Bover, Taltavull, Gastaldo, Luengo, Izquierdo, Juando-Prats, Sáenz de Ormijana \& Robledo, 2015; Briones Vozmediano, Agudelo Suárez, López Jacob, Vives Cases, Ballester Laguna \& Ronda Pérez, 2014; Canga, Vivar \& Naval, 2011; Casado Marín, 2006; Casado-Mejía et al., 2009; Galiana-Gómez de Cádiz et al., 2008; Gallart, Cruz \& Zabalegui, 2013; Garcés, Carretero, Ródenas \& Vivancos, 2010; Zabalegui et al., 2008), Italia (ANS, 2015; INPS, 2015; Lazzarini, Santagati \& Bollani, 2007; Melchiori, 2008; Starovici, 2015), Reino Unido (Bunting \& Jenkins, 2016; Davis \& Smith, 2013; Downs \& Collins, 2015; Gu, Rosenberg \& Zeng, 2017; Hawkes et al., 2015; Henderson, Baker \&Mak, 2016; Likupe, 2014; Meeuwesen, Harmsen, Bernsen \& Bruiknzeels, 2006; Mold, Fitzpatrick \& Roberts, 2005; Sienko, 2002; Slewa-Younana, Santalucia, McDonald \& Salem, 2016; Victor, Martin \& Zubair, 2011), Rumanía (Ministry of Labour, 2017; National Council of Elderly People, 2015; The Government of Romania, 2015).

El análisis de necesidades, desde nuestra doble perspectiva de partida (necesidades de cuidado para las personas mayores y necesidades formativas para sus cuidadores informales), puede sintetizarse en seis aspectos clave: 1) Soledad, 2) Problemas de comunicación, 3) Necesidad de un cui- 
dado centrado en la persona y culturalmente apropiado, 4) Limitación de la Movilidad, 5) Seguridad y Protección (aspectos éticos y legales del cuidado), 6) Recursos sociosanitarios.

\subsection{Soledad}

La literatura científica destaca el problema de la soledad asociada a las personas mayores que residen solas en casa o institucionalizadas en centros. Dicha soledad es a menudo la consecuencia del aislamiento, en particular para aquellos que viven solos. También es resultado de la pérdida (cónyuge) y la tristeza. Otro factor determinante son las dificultades para la comunicación, cuyas causas resultan múltiples: la barrera del idioma con su cuidador, pérdida de memoria, otros problemas de salud.

Para atender esta necesidad de cuidado, la literatura destaca la necesidad formativa del cuidador para ser capaz de localizar y acceder a información y servicios que puedan ayudar a aliviar la soledad de la persona mayor.

\subsection{Problemas de comunicación}

Las diferencias en el idioma entre la persona mayor y su cuidador se muestra como uno de los grandes retos a abordar. Parece deseable que los cuidadores inmigrantes y extranjeros posean un mínimo de competencia oral y escrita en el idioma de la persona atendida.

Igualmente se hace referencia a la importancia de la comunicación intercultural para un cuidado seguro y compasivo proporcionado por/para personas de procedencias culturales diversas.

Finalmente, la comunicación con la persona con demencia es otro reto inequívocamente destacado en las fuentes consultadas.

\subsection{Necesidad de un cuidado centrado en la persona y culturalmente apropiado}

Si bien la literatura científica admite la importancia del papel de la familia en el cuidado de las personas mayores, igualmente muestra un retrato realista de la situación actual en la que, debido a Ios anteriormente citados cambios sociales y demográficos, muchas familias encuentran grandes dificultades para atender a sus seres queridos mayores, tornando en muchos casos en una responsabilidad imposible de asumir.

Existe consenso sobre la necesidad de que los cuidadores sean formados para comprender el contexto cultural de la persona mayor, siendo capaces de evaluar sus necesidades de forma culturalmente sensible, desarrollando una labor de cuidado compasivo que atienda tales necesidades, respetando sus valores culturales, creencias, costumbres, tradiciones, y creencias y prácticas religiosas.

\subsection{Limitación de la Movilidad}

La limitación de la movilidad constituye un problema común para muchas personas mayores, debido a múltiples causas: fragilidad debida a la edad, problemas de salud tales como artritis, problemas de salud mental tales como depresión, y problemas cognitivos, tales como pérdida de memoria y confusión.

La formación específica en este sentido resulta imprescindible, no sólo para que los cuidadores comprendan los beneficios de la movilidad, sino también para ser capaces de animar y motivar a las personas mayores a las que cuidan hacia un estilo de vida más activo, a través de actividades sencillas en las que las personas mayores puedan participar a diario.

Igualmente, los cuidadores deben conocer los servicios comunitarios que puedan resultar de ayuda para el abordaje de los problemas de movilidad de las personas mayores a las que atienden. 


\title{
NECESIDADES FORMATIVAS DE LOS CUIDADORES INMIGRANTES DE PERSONAS MAYORES EN EL HOGAR EN EUROPA: PROYECTO IENE7
}

\author{
4.5. Seguridad y Protección (aspectos éticos y legales del cuidado) \\ Son varios los aspectos destacados en la literatura científica sobre este particular: \\ -Las responsabilidades legales de los cuidadores y de sus empleadores, que varían en los dife- \\ rentes países europeos. \\ -Las responsabilidades éticas de los cuidadores en el ejercicio de su trabajo, en relación a las \\ personas mayores atendidas y sus familiares. \\ -La prevención del abuso, violencia y discriminación hacia la persona mayor.
}

\subsection{Recursos sociosanitarios}

La comparativa de los resultados de las búsquedas bibliográficas nacionales desarrolladas en paralelo, así como la propia literatura científica, ponen de manifiesto la existencia de importantes diferencias en la atención y recursos disponibles para el cuidado de las personas mayores en los diversos países europeos.

Este aspecto resulta trascendente no sólo para los cuidadores inmigrantes y extranjeros, sino también para las personas mayores migrantes y refugiadas, particularmente para aquellas con limitaciones en el idioma del país de acogida y/o de llegada reciente al mismo.

\section{CONCLUSIONES}

El proyecto europeo IENE7 surge con el objetivo general de ofrecer formación para los cuidadores informales, mejorar sus oportunidades de trabajo, fortalecer su posición en el mercado laboral y aumentar la calidad en la atención a los mayores en diversos países europeos. Nuestra propuesta aborda principalmente a los grupos desfavorecidos de inmigrantes, muchos de ellos no cualificados, para mejorar su comunicación profesional, intercultural y social con el fin de aumentar su profesionalidad, empleabilidad, fomentar su participación activa y cambiar las actitudes estigmatizadas de la sociedad y algunas instituciones.

Así, este proyecto desea mejorar el acceso, la participación y el aprendizaje de grupos desfavorecidos, prioridad horizontal de los programas europeos ERASMUS +.

El conocimiento de las necesidades de cuidado para las personas mayores y, en paralelo, las necesidades formativas de sus cuidadores informales, constituye un punto de partida imprescindible para la propuesta y desarrollo de un modelo adecuado de educación dirigido a este colectivo de cuidadores, capaz de dar respuesta a las mismas, objetivo general de nuestro proyecto europeo IENE7, para atender esta importante y urgente demanda social.

\section{REFERENCIAS}

Álvarez, E.R., Elordui, N.L., Errami, M., Rodríguez, A.R., Riguera, C.P., de la Hoz, G.V. \& Márquez, G.M. (2009). Relación del estatus migratorio y del apoyo social con la calidad de vida de los marroquíes en el País Vasco. Gaceta Sanitaria, 23, 29-37. Recuperado de: http://www.sciencedirect.com/science/article/pii/S0213911109003136

ANS. (2015). Anziani e Non Solo. Recuperado de: http://formazione.anzianienonsolo.it/moodle Bover, A., Taltavull, J.M., Gastaldo, D., Luengo, R., Izquierdo, M.D., Juando-Prats, C., Sáenz de Ormijana, A. \& Robledo, J. (2015). Calidad de vida de trabajadoras inmigrantes latinoamericanas como cuidadoras en España. Gaceta Sanitaria, 29(2), 123-126. Recuperado de: http://www.sciencedirect.com/science/article/pii/S0213911114002441

Briones Vozmediano, E., Agudelo Suárez, A.A., López Jacob, M.J., Vives Cases, C., Ballester Laguna, F. \& Ronda Pérez, E. (2014). Percepción de las trabajadoras inmigrantes del servicio doméstico sobre los efectos de la regulación del sector en España. Gaceta sanitaria, 28(2), 109-115. Recuperado de: 
http://www.sciencedirect.com/science/article/pii/S0213911113001398

Bunting, M. \& Jenkins, C. (2016). Transcultural nursing strategies for carers of people with dementia. Nursing Older People, 28(3). Recuperado de: http://dx.doi.org/10.7748/nop.28.3.21.s23

Canga, A., Vivar, C. G., \& Naval, C. (2011). Dependencia y familia cuidadora: Reflexiones para un abordaje familiar. Anales Del Sistema Sanitario de Navarra, 34(3), 463-469. https://doi.org/10.4321/S1137-66272011000300012 Recuperado de: http://scielo.isciii.es/pdf/asisna/v34n3/revision3.pdf

Casado Marín, D. (2006). La Atención a la dependencia en España. Gaceta Sanitaria, 20(Supl 1 (Informe SESPAS 2006)), 135-142. https://doi.org/10.1157/13086037 Recuperado de: http://www.aes.es/Noticias/wpcasado.pdf

Casado-Mejía, R., Ruiz-Arias, E., \& Solano-Parés, A. (2009). Características de la producción científica sobre cuidados familiares prestados por mujeres inmigrantes. Gaceta Sanitaria, 23(4), 335-341. https://doi.org/10.1016/j.gaceta.2009.03.005 Recuperado de: http://scielo.isciii.es/pdf/gs/v23n4/revision2.pdf

Davis, B. \& Smith, M. (2013). Developing Culturally Diverse Direct Caregivers for Care Work with Older Adults: Challenges and Potential Strategies. The Journal of Continuing Education in Nursing, 44(1), 22-30. Recuperado de: http://dx.doi.org/10.3928/00220124-20121101-54

Downs, M. \& Collins, L. (2015). Person-centred communication in dementia care. Nursing Standard, 30(11). Recuperado de: http://dx.doi.org/10.7748/ns.30.11.37.s45

European Commission (2009). 2009 Ageing Report: Economic and budgetary projections for the EU-27 Member States (2008-2060). Recuperado de: http://ec.europa.eu/economy_finance/publications/pages/publication14992_en.pdf

Eurostat (2016). Projected old-age dependency ratio. Recuperado de: http://ec.europa.eu/eurostat/tgm/table.do?tab=table\&init=1\&language=en \&pcode=tsdde511\&plugin=1

Galiana-Gómez de Cádiz, M. J., de la Cuesta-Benjumea, C., \& Donet-Montagut, T. (2008). Cuidadoras inmigrantes: características del cuidado que prestan a la dependencia. Enfermería Clínica, 18(5), 269-272. https://doi.org/10.1016/S1130-8621(08)72386-6. Recuperado de: http://www.elsevier.es/es-revista-enfermeria-clinica-35-linkresolver-cuidadoras-inmigrantescaracteristicas-del-cuidado-S1130862108723866

Gallart A., Cruz F. \& Zabalegui A. (2013). Factors influencing burden among non-professional immigrant caregivers: a case-control study. Journal of Advanced Nursing 69(3), 642-654. doi: 10.1111/j.1365-2648.2012.06049.x Recuperado de: http://onlinelibrary.wiley.com/doi/10.1111/j.1365-2648.2012.06049.x/epdf

Garcés, J., Carretero, S., Ródenas, F., \& Vivancos, M. (2010). The care of the informal caregiver's burden by the Spanish public system of social welfare: A review. Archives of Gerontology and Geriatrics, 50(3), 250-253. https://doi.org/10.1016/j.archger.2009.04.011 Recuperado de: http://www.sciencedirect.com/science/article/pii/S0167494309000971?via\%3Dihub

Gu, L., Rosenberg, M. \& Zeng, J. (2017). Changing caregiving relationships for older home-based Chinese people in a transitional stage: Trends, factors and policy implications. Archives of Gerontology and Geriatrics, 70, 219-229. Recuperado de: http://dx.doi.org/10.1016/j.archger.2017.02.002

Hawkes, D., Hingley, D., Wood, S. \& Blackhall, A. (2015). Evaluating the VERA framework for communication. Nursing Standard, 30(2), 44-48. Recuperado de: http://dx.doi.org/10.7748/ns.30.2.44.e10052

Henderson, S., Baker, M. \& Mak, A. (2016). Strategies used by nurses, academics and students to 


\section{NECESIDADES FORMATIVAS DE LOS CUIDADORES INMIGRANTES DE PERSONAS MAYORES EN EL HOGAR EN EUROPA: PROYECTO IENE7}

overcome intercultural communication challenges. Nurse Education in Practice, 16(1), 71-78. Recuperado de: http://dx.doi.org/10.1016/j.nepr.2015.08.010

IENE7 Project (2017). Improving communication, intercultural and social skills for foreigners and migrants who work as caregivers of elderly people in Europe (ID: 2017-1-R001-KA202037272). Recuperado de: https://iene7.eu/project/

INPS. (2015). Badanti: categoria crescita costante. II Sole 24 Ore. Recuperado de: http://www.ilsole24ore.com/art/impresa-e-territori/2017-01-07/badanti-categoria-crescita-costante161711.shtml?uuid=AD68URSC

JIFE (2015). Informe 2014, 1-140. Recuperado de https://www.incb.org/documents/Publications/AnnualReports/AR2014/Spanish/AR_2014_ESP. pdf

Lazzarini, G., Santagati, M. \& Bollani, L. (2007). Prendersi cura in famiglia: il rapporto fra caregiver e anziano non autosufficiente. Tra cura degli altri e cura di sé. Percorsi di inclusione lavorativa e sociale delle assistenti familiari. Ed. FrancoAngeli. Milano.

Ley 39/2006, de 14 de diciembre, de Promocion de la Autonomia Personal y Atencion a las personas en situacion de dependencia. Boletin Oficial del Estado 26(299), 44142-44156.

Recuperado de http://www.boe.es/boe/dias/2006/12/15/pdfs/A44142-44156.pdf

Likupe, G. (2014). Communicating with older ethnic minority patients. Nursing standard, 28(40), 37-43. Recuperado de: https://search.proquest.com/docview/1785259827?accountid=12441

Meeuwesen, L., Harmsen, J., Bernsen, R. \& Bruiknzeels, M. (2006). Do Dutch doctors communicate differently with immigrant patients than with Dutch patients? Social Science \& Medicine, 63, 2407-2417. Recuperado de: https://www.sciencedirect.com/science/article/pii/S027795360600308X

Melchiori, C. (2008). Assistere l'anziano a domicilio: il ruolo per la badante. $9^{\text {th }}$ Nursing Multifunctional Course. Recuperado de: http://www.sigg.it/diapositive53/nursing/Melchiori.pdf

Ministry of Labour. (2017). Classification of Occupations in Romania, Group 5 Service workers, pp. 9-10. Recuperado de:

http://www.mmuncii.ro/j33/images/Documente/MMJS/Munca/COR/2017-04-06-2Grupa_Majora_5.pdf

Mold, F., Fitzpatrick, J. \& Roberts, J. (2005). Caring for minority ethnic older people in nursing care homes. British Journal of Nursing, 4 (11), 601-606. Recuperado de: https://search.proquest.com/docview/199498329?accountid=12441

Moreno-Cámara, S., Palomino-Moral, P. Á., Moral-Fernández, L., Frías-Osuna, A., \& Del-PinoCasado, R. (2016). Problemas en el proceso de adaptación a los cambios en personas cuidadoras familiares de mayores con demencia. Gaceta Sanitaria, 30(3), 201-207. doi: 10.1016/j.gaceta.2016.02.004

National Council of Elderly People. (2015). Social assistance for the elderly. Recuperado de: http://www.cnpv.ro/index.php/analize/analize-si-studii-elaborate-2015

Navalpotro, S., González, E., Martín, S., Vicente, M. J., Comet, P., \& Vidal, C. (2006). Oferta de servicios sociosanitarios en Atención Primaria para la atención a mayores de 65 años. Gerokomos, 17(4), 189-196.

Notivol Tejero, P., Pollan Rufo, M., \& Gabari Gambarte, M. I. (2004). Percepción de cuidadoras inmigrantes extranjeras. Sobre el cuidado, genérico y profesional enfermero, al anciano en domicilio. Huarte de San Juan. Psicología, (11), 153-171.

Rodríguez Rodríguez, V., Martín Coppola, E., Marcu, S., Ramos Espina, S., Rojo Pérez, F., Fernández-Mayoralas, G., .. \& Rogero García, J. (2010). Inmigración y cuidados de mayores 
en los hogares de la Comunidad de Madrid. Informes Portal Mayores, (102), 1-641.

Recuperado de http://www.imsersomayores.csic.es/documentos/documentos/rodriguez-inmigracion-01.pdf

Sienko, K. (2002). Providing culturally competent health services for ethnic minorities. Nursing Standard, 16(48). Recuperado de: https://search.proquest.com/docview/219817973?accountid $=12441$

Slewa- Younana, S., Santalucia, Y., McDonald, R. \& Salem, M. (2016). Enhancing the lives of older refugees: an evaluation of a training resource. International Journal of Mental Health Systems, 10(36). Recuperado de: http://dx.doi.org/10.1186/s13033-016-0067-5

Starovici, M.M. (2015). Prendersi cura di chi si prende cura. Recuperado de: http://tesi.cab.unipd.it/51192/1/starovici.marioaramary.1046243.pdf.

The Government of Romania. (2000). The National Grid for Assessing the Needs of the Elderly. Government DECISION no. 886 of October 5, 2000, for the approval of the National Grid for Assessing the Needs of the Elderly. Recuperado de: http://www.mmuncii.ro/pub/imagemanager/images/file/Legislatie/HOTARARI-DE-GUVERN/HG886-2000.pdf

Victor, C., Martin, W. \& Zubair, M. (2011). Families and caring amongst older people in South Asian communities in the UK: a pilot study. European Journal of Social Work, 15(1), 81-96. Recuperado de: http://dx.doi.org/10.1080/13691457.2011.573913

Zabalegui Yárnoz, A., Navarro Díez, M., Cabrera Torres, E., Gallart Fernández-Puebla, A., Bardallo Porras, D., Rodríguez Higueras, E., ...\& Argemí Remon, J. (2008). Eficacia de las intervenciones dirigidas a cuidadores principales de personas dependientes mayores de 65 años. Una revisión sistemática. Revista Española de Geriatría Y Gerontología, 43(3), 157-166. https://doi.org/10.1016/S0211-139X(08)71176-4 Recuperado de: http://www.elsevier.es/esrevista-revista-espanola-geriatria-gerontologia-124-articulo-eficacia-las-intervenciones-dirigidas-cuidadores-S0211139X08711764 
